\title{
BMJ Open Acute pain management among patients with opioid maintenance therapy: specificities and difficulties identified in primary care: a qualitative study
}

\author{
Morgane Guillou Landreat (D , ,,2 Melia Baillot, ${ }^{3}$ Delphine Le Goff, ${ }^{1}$ \\ Jean Yves Le Reste ${ }^{4}$
}

To cite: Guillou Landreat M, Baillot M, Le Goff D, et al. Acute pain management among patients with opioid maintenance therapy: specificities and difficulties identified in primary care: a qualitative study. BMJ Open 2021;11:e044433. doi:10.1136/ bmjopen-2020-044433

- Prepublication history for this paper is available online. To view these files, please visit the journal online (http://dx.doi. org/10.1136/bmjopen-2020044433).

Received 07 September 2020 Revised 25 November 2020 Accepted 18 December 2020

Check for updates

(c) Author(s) (or their employer(s)) 2021. Re-use permitted under CC BY-NC. No commercial re-use. See rights and permissions. Published by BMJ.

${ }^{1}$ EA SPURBO, Universite de Bretagne Occidentale, Brest, France

2addictologie, CHRU de Brest, Brest, France

${ }^{3}$ EA SPURBO, UBO, Brest, France ${ }^{4}$ ERCR SPURBO, Universite de Bretagne 0ccidentale, Brest, France

Correspondence to Dr Morgane Guillou Landreat; morgane.guillou@chu-brest.fr

\section{ABSTRACT}

Objectives In the last 30 years, opioid maintenance treatment prescription (OMT) has changed patients' and also changed physicians' practices. General practitioners (GPs) have to deal with patients on OMT who are in acute pain. The objective of this qualitative study was to explore medical care challenges and solutions identified by GPs in the management of acute pain among patients receiving OMT.

Design and setting Qualitative study with semistructured interviews were used as a data collection technique with a sampling strategy using a snowball sampling method to obtain a purposive sample of practicing GPs. Analysis was undertaken using a thematic analysis method.

Participants Twelve GPs, working in France (Brittany) who prescribe OMT were interviewed.

Results The thematic analysis resulted in two main themes relating to specificities and difficulties identified: (1) Medical care and training challenges identified by GPs treating patients on OMT with acute pain, with four subthemes : management of these situations not concerning primary care, lack of training prompts GPS to rely on peer and specialist support, lack of guidelines and conflicting recommendations between clinicians in different settings (2) linked to the patient-GP relationship, with six subthemes: Implementing an individualised centred approach, acute pain management during OMT relies on a relationship based on trust, GPs found difficulties in evaluating and treating pain, difficulties in care adherence, fear of patients destabilisation, fear of misuse and diversion.

Conclusion The complexity of acute pain and OMT entails significant challenges for clinicians and patients. In primary care, it is hard to achieve a balance between pain relief and opioid use disorder treatment, in a global patient-centred approach. Fear of misuse or diversion was not a important factor, except for patients not known to the practitioners, but GPs were concerned with the risks of patient destabilisation in situations of acute pain.

\section{BACKGROUND}

Opioid dependence, whether as a result of taking licit or illicit opioids, is a major public health issue worldwide. Opioid dependence accounts for about 15479000 individuals
Strengths and limitations of this study

- This is the first study exploring the specificities and the difficulties identified by general practitioners (GPs) in the management of acute pain among patients receiving opioid maintenance treatment (OMT).

- A validated qualitative approach (thematic analysis) helped to analyse the rich and complex data, and to obtain in-depth answers about specificities and difficulties of acute pain management in patients with an OMT in primary care.

- In primary care, in a global patient-centred approach, it is hard to achieve a balance between pain relief and opioid users disorders treatment.

- The selected sample of GPs limits transferability of the data.

around the world. ${ }^{1}$ In Europe, the mean prevalence of opioid users disorders (OUD), defined according the Diagnostic and statistical Manual of Mental Disorders (DSM 5), ${ }^{2}$ is estimated to be between 3.6 and 4.4 per 1000 inhabitants (15-64 years of age). To treat OUD, opioid maintenance treatment (OMTs) such as methadone, buprenorphine or buprenorphine/naloxone have amply demonstrated their effectiveness. ${ }^{3} 4$ This system has proved to be efficient in terms of public health, access to care and risk reduction. ${ }^{5-7}$ In France, 170000 patients are currently being treated for OUD, of whom $65 \%$ are on BHD and $35 \%$ on methadone. ${ }^{8}$ OMT is a long-term treatment, sometimes maintained for life.

The population of patients under OMT prescribed for an opioid use disorder requires specific care: opioid-dependent patients present more comorbidities than the general population, and have an especially heightened susceptibility to acute pain. First, opioid-dependent subjects present medical 
histories with higher risks of infectious diseases, ${ }^{9-11}$ traumatic events, medical complications, such as serious cardiac abnormalities and a higher rate of epileptic seizures, ${ }^{12-15}$ liver disease and chronic pain. ${ }^{16}{ }^{17}$ Second, they present psychological vulnerabilities, such as depression and anxiety, that may be associated with a higher risk factor for pain and these are also morbidity and mortality risk factors. ${ }^{18}$ Third, pharmacologically, two phenomena can lead to more difficult management of acute pain in opiate-dependent patients: opioid tolerance and opioid hyperalgesia. ${ }^{19-21}$ These two phenomena lead to a higher susceptibility to pain among opioid-dependent subjects. The pain intolerance of patients receiving OMT can be conceptualised as a latent hyperalgesia secondary to longterm opioid exposure. ${ }^{22}$ As a consequence, pain management among patients with an OMT requires significantly higher doses of pain medication. ${ }^{23}$

Pain management among patients with an OMT is defined as a challenging situation. Patients in this situation at admission to hospital fear opioid withdrawal, pain, discrimination and relapse. ${ }^{24}$ For their part, clinicians are unwilling to trust patients with an OUD, they do not trust reports on pain which could be enhanced to obtain opioids and the resulting euphoria, they are aware of the risk of opioid diversion, the risk that the patient might leave the hospital against medical advice, and the medical risk of overtreatment, leading to opioid-induced ventilatory impairment. ${ }^{24}$ Guidelines exist for the management of opioid use disorder but very few address the specific needs, such as those related to pain, of populations of patients with on OMT. The American Society of Addiction Medicine (ASAM) edited practice guidelines for the Use of Medications in the Treatment of Addiction Involving Opioid Use and pain, ${ }^{25}$ and even if evidencebased strategies for pain management among patients receiving OMT are limited, a few studies have defined strategies. ${ }^{242627}$ The ASAM practice guideline states that pharmacotherapy in conjunction with psychotherapy should be considered for patients with pain and an OUD. Moreover when OMT is prescribed, OMT dosage for pain should be maintained or increased on a temporary basis and administered in a staggered schedule rather than as a single daily dose, and non-narcotic medications, like acetaminophen or Non steroid antiflammatory Drugs (NSAIDs) should be tried initially. ${ }^{25}$

All these previous studies and guidelines focused on specialised care in hospitalisation, but OMT, like acute pain situations, are mainly managed in primary care, and more particularly in France. Indeed, methadone marketed in 1995 and buprenorphine in 1996 are both available in France as part of a global therapeutic strategy. BHD can be prescribed by any physician, MTD primary prescription is restricted to physicians operating in specialised units or hospitals, but after a period of stabilisation, follow-up and prescription can be carried out by any physician. ${ }^{428}$

We hypothesised that general practitioners (GPs) who prescribe OMT are frequently exposed to situations of acute pain management for patients with a regular treatment of OMT prescribed for an OUD. The literature is sparse about acute pain management among patients on OMT, especially in primary care. Consequently, the present objective of this qualitative study was to explore medical care challenges and solutions identified by GPs in the management of acute pain among patients receiving OMT.

\section{METHODS}

A research committee including GPs with experience in qualitative research (MD, MD-PhD), a psychiatrist specialised in addictive disorders (MD, $\mathrm{PhD}$ ) and methodologists (PhD) was formed. A qualitative design consisting in semi-structured face-to-face interviews was used in this study. The research committee (all trained and experienced in qualitative research) designed a qualitative interview guide consisting of six open-ended questions. This guide was tested on a sample of GPs to ensure that the duration of the interview was maximum half an hour. The guide was intentionally short to enable the participation of GPs on this complex topic. The qualitative method with face-to-face interviews is considered as ideal when in-depth description of caregiver or healthcare professional experiences in relation to a particular phenomenon is desired. A qualitative approach was chosen because this type of research could enable us to explore the specificities and the difficulties in management of this complex situation by GPs. ${ }^{29}$ Interviews were chosen because we wanted to access to the personal experience of each GP, and not that of the group of GPs as a whole. We aimed to pinpoint issues that had not been documented previously. ${ }^{30}$

\section{Sampling and recruitment}

GPs were recruited from a network of GPs practising in the administrative region of this study (Finistere, Brittany, France) with professional experience of OMT management. The main selection criterion was experience with OMT prescribing in primary care (number of patients on OMT that they followed), and also the numbers of years of practice in primary care, and the location of practice (urban, rural, semirural). The sampling strategy was implemented in two steps to obtain a purposive sample. In the first step, three GPs were recruited from the network, they were identified as OMT prescribers by the study research committee. In the second stage, starting from these first three GPs, a snowballing method was used to recruit new GPs on the basis of their personal experience in OMT management. Data collection continued until theoretical saturation was reached, whereby no new themes emerged from the data collected. ${ }^{31}$ No-one declined because of lack of interest in the study. Written informed consent was obtained from all the participants in this study. 


\section{Data collection}

Participants were contacted for the study by phone, they were told that the study concerned one single face-toface interview on the management of OMT, and that it was research work led by the department of primary care in the University of Brest (EA SPURBO 7479). A GP researcher (the second author of this article: MD, female and trained in qualitative studies) interviewed all the participants in the study between 24 April and 25 June 2015. No relationship was established between the researcher and the selected GPs prior to the start of the study. The study was the researcher's only occupation during inclusion and the analysis of the data. The interviewer decided to work on this topic because she was herself, as a GP, confronted with difficulties in medical practice in managing acute pain for patients on OMT in primary care. She noted a lack of information in the literature, and in collaboration with the coauthors it was decided to design this study. Individual face-to-face semistructured interviews were carried out with GPs, in their workplace, according to the GP preferences and availabilities, without any other participant. A semistructured interview guide was developed based on the study objectives to guide the interviewer and ensure uniformity. It was tested on a small sample of GPs by the interviewers before the study. The guide covered the following areas : confirmation of consent, GP characteristics (age, gender, number of years of practice, type of practice, experience with OMT) and questions regarding the study objective (table 1): experience with OMT and acute pain, specificities and difficulties in the management of these situations. GPs were also provided with the opportunity to talk about any issue related to acute pain management among patients on OMT that was not covered by the questions.

All the interviews were audiorecorded and the verbatim was transcribed and checked by the first and second authors. No fields notes were made during the interview. In order to improve validity and credibility, all transcripts were returned to participants for checking, none made any comments.

The duration of the interview was between 5 and $47 \mathrm{~min}$ $($ median $=20 \mathrm{~min})$.
The participants provided their written informed consent to participate in this study. The ethics committee approved this consent procedure.

\section{Patient and public involvement}

No patient or public were involved in the design, or conduct or reporting or dissemination plans of the study.

\section{Data analysis}

A thematic analysis, based on a phenomenological interpretative approach, was conducted. ${ }^{32}$ It allows the researcher to explore the experience of participants, the meaning they give to their experience and the underlying psychological mechanisms of all the interviews. ${ }^{33} \mathrm{~A}$ six-step process was used to guide the data analysis. Each interview was transcribed in verbatim and checked by the first and second authors, and both perused the data twice to help them familiarise with the data. Line-by-line manual data coding was used to code the individual transcripts, with an open coding method. The open coding was independently checked by the two members of the research committee experienced in qualitative research (JYLR, DLG). Duplicate codes were removed and the different codes were sorted into potential themes. All relevant codes extracted from the data were collated into different themes. When a set of candidate themes was obtained, the refinement of these themes started. Thematic maps were constructed and implemented. The themes were named and the final report was written. Peer review/debriefing in the research committee were used to enhance the thoroughness and reliability of the study findings. ${ }^{32}$

\section{RESULTS \\ Participants}

Twelve GPs were interviewed (Finistère region, Brittany, France) in 2015. The social and professional profiles of the GPs are presented in table 2.

Following a six-step thematic analysis process, emerging themes were classified under two main themes corresponding to the specificities and the difficulties of

Table 1 Interview guide

\begin{tabular}{|c|c|}
\hline Aim & To explore how GPs manage acute pain in patients with an OMT \\
\hline Ice breaking question & How many patients with an OMT do you regularly follow? \\
\hline \multirow[t]{5}{*}{ Questions for taking the discussion further } & $\begin{array}{l}\text { Could you tell us about the last patient on OMT you saw who was suffering from } \\
\text { pain? }\end{array}$ \\
\hline & What are your thoughts about the pain experienced by patients on OMT? \\
\hline & $\begin{array}{l}\text { Which difficulties do you encounter when evaluating the pain experienced by } \\
\text { patients on OMT? }\end{array}$ \\
\hline & Do you change your pain management when patients are receiving OMT? \\
\hline & How do you feel about this type of patient consultation? \\
\hline Reopening question & Do you have anything further to add on this topic? \\
\hline
\end{tabular}

GP, general practitioner; OMT, opioid maintenance treatment. 
Table 2 Participants interviewed

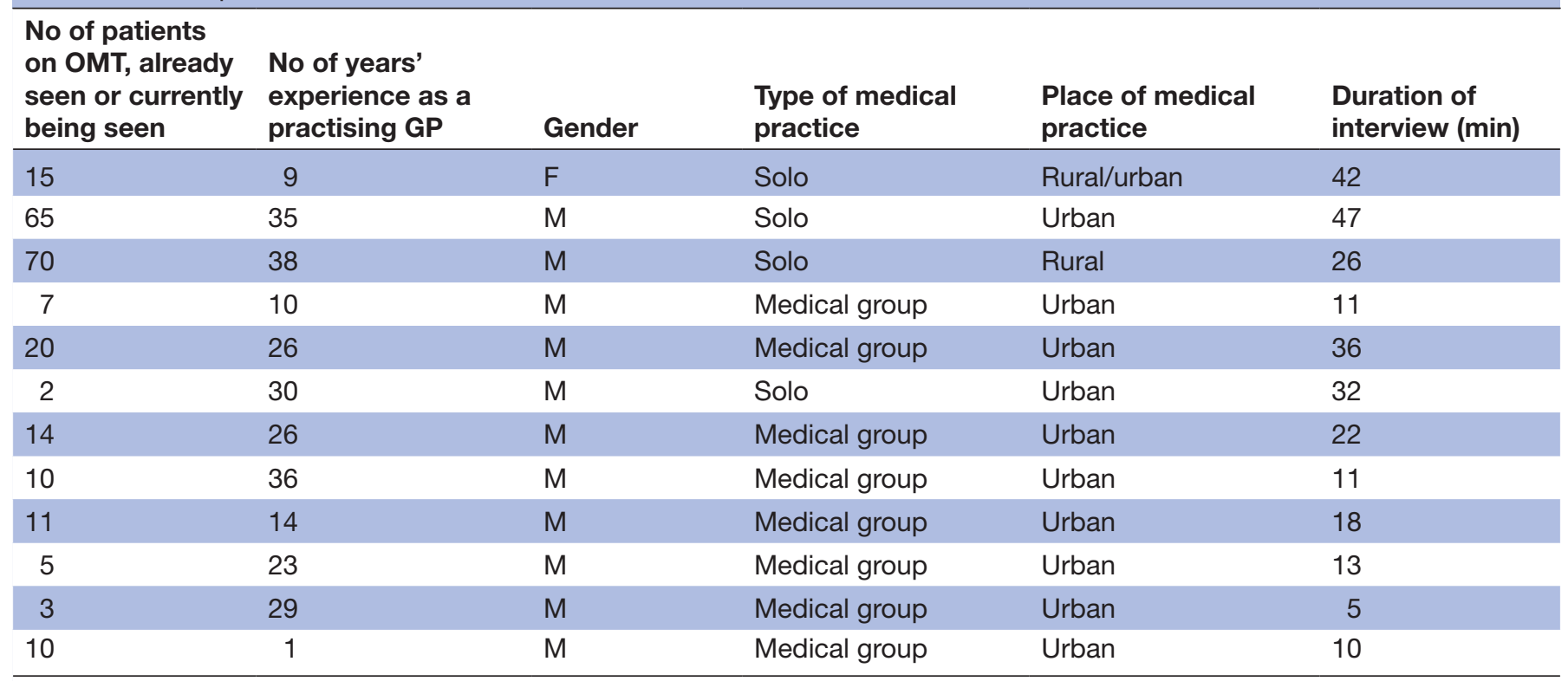

F, female; GP, general practitioner; M, male; OMT, opioid maintenance treatment.

management of acute pain among patients on OMT in primary care: themes linked to medical skills and themes linked to the patient-GP relationship. Subthemes within each of these themes are described below. The themes and subthemes are collated in figure 1.

As it was impossible to describe all the qualitative data, only the main themes and subthemes are described. Where the themes and subthemes that emerged were described in detail, they were illustrated by selected verbatim drawn from all the interviews involved. Verbatim accounts are in italics.

\section{Medical care and training challenges identified by GPs treating patients on OMT with acute pain}

This first theme corresponds to the specificities and difficulties that GPs perceived, and that could be linked to their professional experience, their perception of their professional missions, their training and professional supports as guidelines or networks. In this theme, four subthemes were identified : the management of acute pain in patients with an OMT was considered as not concerning primary care, they identified a lack of training prompts GPs to rely on peer and specialist support, lack of guidelines and conflicting recommendations between clinicians in different settings.

\section{Management of these situations does not concern primary care}

GPs interviewed in this study thought that the level of care needed for these patients was above and beyond what was regularly provided in primary care. GPs expressed anxiety about treating patients on OMT who needed a level of complexity of care, but often felt that they were the patients' only option.

The GPs found that being confronted with intense pain was a rare situation 'really intense, acute pain,
(...), we don't necessarily see it; people will have done something about it. They will have called (...) they will have called an ambulance, they will have gone to A\&E'E5-1.158-165). The GPs reported that as they were not particularly involved with opiate-dependent patients they did not need to spend time in training on this subject 'it is more (...) for colleagues who are much more involved, who have the time to get training in the field of drug addiction care in particular, because the problem for us is that we have a wide range of problems to deal with' (E5-1.291-295).

Nevertheless they deplored the lack of time available to manage these situations: "we don't have an hour ahead of us, you see, you have to understand that an appointment in family practice lasts 15 minutes, for which 25 euros are reimbursed by social security, that's basically the average, okay? So, in 15 minutes we will have everything to deal with, so that's what you have to keep in mind, and that's the problem in general practice' (E5-1.185-192).

Finally, the GPs described the difficulty in providing care in response to a patient's request to be treated solely by their GP: 'And often the difficulty arises from the fact that, precisely because they are causing you problems and you feel your competence is being stretched to its limit in caring for them, they do not want to leave you... you are their only contact and you remain so, so you have to solve things that are sometimes much more complex than you would like' (E5-1.598-607).

Lack of training prompts GPs to rely on peer and specialist support GPs interviewed in this study thought that their training was limited and that the lack of time also made the management of these cases complex. They relied on peers and/ or on specialists for difficult therapeutic situations. 


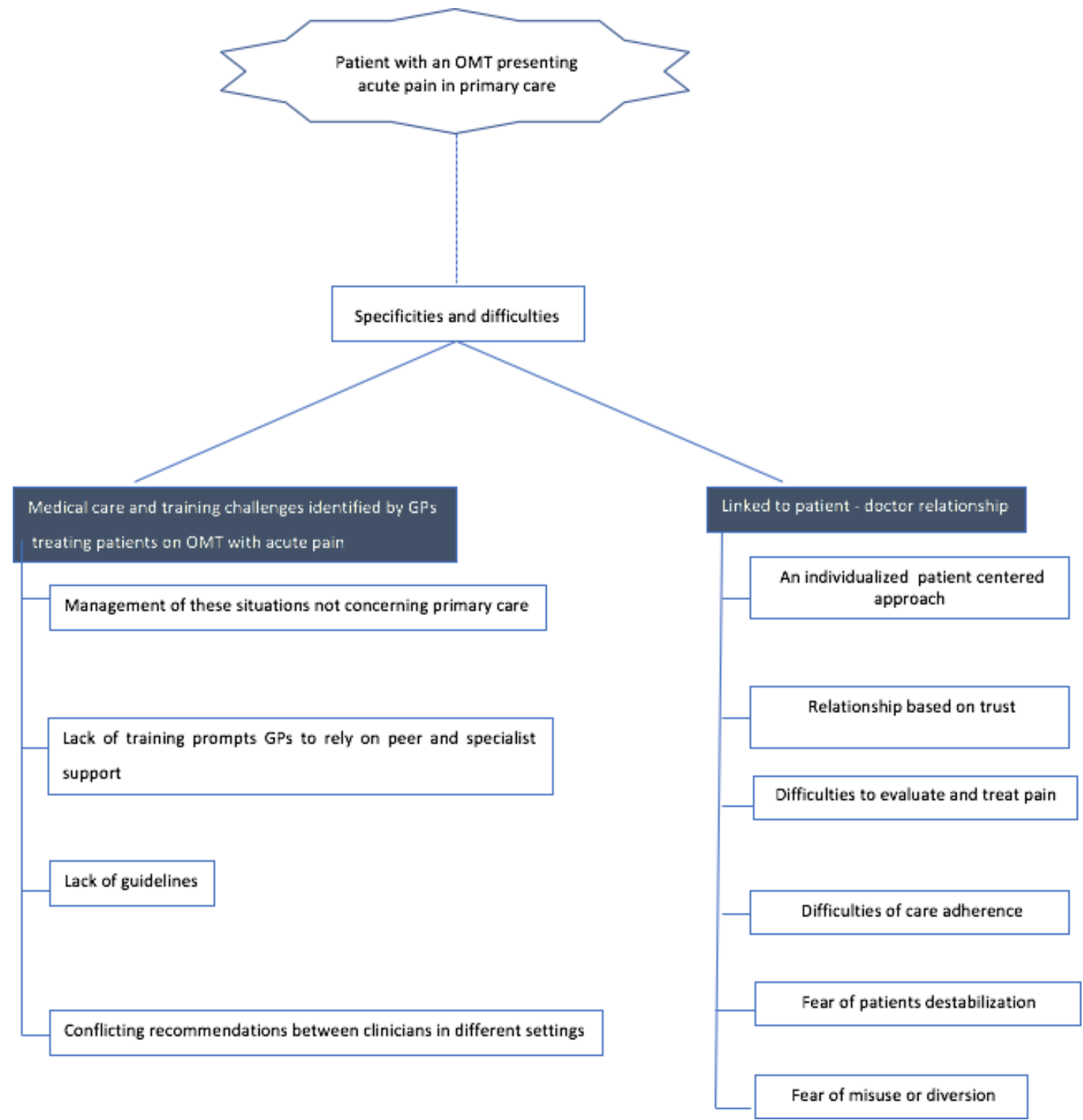

Figure 1 Specificities and difficulties for management of acute pain among patients under OMT in primary care-thematic analyses.GP, general practitioner; OMT, opioid maintenance treatment.

Many of the GPs reported lack of time for training. Some GPs identified inadequate training on the subject 'I think we lack training on this subject'(E5-1.275-278) and they described the desire for specific training to develop their knowledge about OMT and pain management. 'I would be ready to learn more on this topic'(E2-1.784). On the other hand, other respondents were at ease with pain management for patients on OMT: 'I'm not a pain specialist at all, but it's not a problem for me.'(E1-1.546-547).

Many GPs spoke of the need to call on colleagues in difficult therapeutic situations. They first mentioned their colleagues in the same general practice: 'the fractionation of buprenorphine dosage which I had already had to deal with, but then I found, after discussing it with colleagues, that they would have done the same'(E5-1.482-483). They also mentioned the use of addiction networks: 'the centre for drugs, of course, for the whole aspect of toxic sideeffectives and drug treatment' pain centres 'the' Cavale Blanche' pain centres, and the maritime hospital, are quite able to help us with this issue'(E7-1.482-483) and hospitalisation 'if the pain is so severe that she cannot sleep, well I hospitalise her' (E6-1.292)

\section{Lack of guidelines}

GPs interviewed in this study reported a lack of clear guidelines for the management of these complex situations in primary care, and they resorted empiricism from their personal experience of OMT management.

Guidelines for OMT management differ significantly, according to GPs. Some envisage pain management using WHO levels (WHO, 1997) 'I comply with the dosage (...) whatever the medication: doliprane (...) codeine, tramadol and, and opiates' (E2-1.92-94). Most of the GPs reported having no reference recommendations. They reported a lack of consensual data on this subject: 'There is a lack of consensual attitudes concerning acute pain management for patients on OMT' (E5-1. 713-722). 
With regard to prescribing analgesics, a wide variety of practices were described by the physicians: Nonopioid painkiller analgesics (paracetamol) were the most frequently cited. Weak opioids combined with OMT were widely used by some doctors, and not at all by others: 'I felt that pharmacologically it could not work, that we would not have a satisfactory result, because of the competition from substitution treatments, so with the receptors being saturated, it did not seem appropriate to me'(E5-1.417-425). Strong opioid painkillers were also described in various ways: Many of the GPs interviewed avoided this prescription. In contrast, there were others who used morphine in combination with OMT, while, one reported using lower doses. Others still preferred to stop BHD before prescribing morphine. As mentioned above, the GPs also prescribed multimodal analgesia, with extensive use of anti-inflammatory drugs, and sometimes antidepressants, for neuropathic pain.

Regardless of their prescriptions, the GPs stressed the importance of using a second category of medication for analgesic treatment. The reasons given were:

Clarification of the indications: 'I prefer to prescribe analgesics, ultimately, I prefer to dissociate the use of analgesics for analgesic purposes from a specific analgesic used for withdrawal, (...) buprenorphine retains its status as a substitution treatment'. (E9-1. 95-105)'

Clarification of the provisional aspect of this prescription: 'the fact that a (...) second molecule (...) has been prescribed indicates the provisional nature of the treatment'. (E2-1.107-109)

Some physicians described prescribing OMT dosages staggered during the day, with or without an increase in daily dosage. Others associated a non-opioid painkiller or a weak opioid analgesic with OMT: 'When you have to give them morphine, to be sure that methadone is effective I divide it in 2, and then I add either tramadol, or Acupan, or another level 2 medication, avoiding level 3 analgesics' (E3-1.145-150).

Finally, opinions were spread through the day in relation to the effectiveness of codeine. Some physicians described variable patient feedback. Others said they had noted the efficacy of codeine: 'So it's true that, pharmacologically, it shouldn't work, except that it can actually work' (E1-1.47-48). In addition, they did not observe withdrawal syndrome when combining codeine and buprenorphine: 'In my daily professional life, I have never seen codeine-Subutex withdrawal, never, ever...'(E1-1.757-759).

However, many GPs mentioned their reluctance to spread OMT intake for analgesic purposes. They reported difficulties in arguing for the splitting of the OMT in case of acute pain. Indeed, in their opinion this advice is not compatible with their usual efforts which are centred on maintaining once daily doses of OMT, as recommended.: 'That's what is so complicated, explaining to them (patients) that all the medication must be taken in one dose...'(E1-1.275-276).

They resorted to empiricism. Many mentioned that pain management in patients on OMT was founded on their personal experience of treatment management 'I've developed my own methods, I've done things this way, that's how it works' (E2-1.785-786) and for communication management 'now I know a lot about it, I know how to talk to them' (E1-1.157-158).

\section{Conflicting recommendations between clinicians in different settings}

GPs interviewed in this study reported a lack of multidisciplinary network, a lack of availability of the network, and conflicts around management of these situations.

The GPs reported on pain management that was not tailored to the patients during their hospitalisation. This concerned pharmacological treatment and meant that GPs needed to change the treatment after the patient had been discharged: 'The pain management had not been satisfactory $(\ldots)$ in the hospital for example (...) so we had to review the whole scheme'(E5-1.260-273). Making medication changes is hard for GPs because of the sanctity of hospital doctors' prescriptions vs GPs' prescriptions. Moreover, 'f something has been said to a patient in hospital and the family physician starts to contradict what the hospital has said, it makes management difficult.' (E5-1.340-343).

Relationships with pharmacists were also defined as difficult, with conflicting information between GPs and pharmacists, 'Comments are inconsistent, and ultimately run counter to good care'.(E5-1.542-545). However, they stressed the importance of a professional network and reported informal and evolving networks.

The limits of networking were availability of the network, and the negative perceptions that some professionals might hold of opioid-dependent patients: 'Clichés (...) 'the addict is manipulative, (...) he is addicted for the rest of his life', (...) You can't trust him' (E5-1. 687-691).

\section{Specificities and difficulties linked to the patient-GP relationship}

This second theme corresponds to the specificities and difficulties that GPs perceived, and that could be linked to the relationships and more particularly the patient and GP relationship. Six subthemes were identified : first the GPs described an individualised centred approach of patients, they also identified that relationship with patients was based on trust, but they also underlined difficulties to evaluate and treat pain, with difficulties for patients with an OMT in care adherence, with a fear of patients destabilisation and a fear of opioid misuse or diversion.

\section{An individualised patient-centred approach}

GPs interviewed in this study underlined that the management of pain for patients on OMT were integrated in a global management of patients, in a patient-centred approach.

This idea was in line with that of the overall management of the patient in general medicine: 'The patient who comes to me I take as a whole person, not just as a 
drug addict, but as a patient, and so a patient on substitution treatment can also have toothache, (...) or chronic bronchitis,)... (or hypertension...(E5-1.232-243). They described a global relationship between patient and GP 'it is better to know the person, his family, (...) to discuss the weather and things of life ... simply ...(...) (E7-1.359-368)

The GPs reported certain specificities of the population on OMT, they agreed that this is quite a young patient population: 'They are often patients who are still quite young' (E6-1.57). They also mentioned patients with complex psychological profiles: 'a particular psychological profile'(E8-1.72). For pain evaluation, the specificities of patients on OMT were taken in account : 1 . The reduced pain threshold: 'as they have a slightly lower threshold (...) it could possibly be misleading (...) for pain rated higher than 5 (scores range from 0 to 10)'(E1$1.670-673)$; 2. The self-regulation of OMT by patients limits scope for pain evaluation; 3 . The context of psychological suffering: 'Often there is a psychological component (...) anxiety, stress, (...) between psychological pain and nociceptive pain (...) the boundary between the two is not always easy to determine' (E12-1.84-91). One GP also mentioned patients having identified different perceptions of their own body compared with the general population: 'They have notions about their own body as not being the same as other people's' (E6-1.99-100).

They mentioned the health and lifestyle advice they provided to reduce pain when they saw patients. They also described the importance of supportive psychotherapy: 'I am one of the old-time GPs who think, yes, that conversation has therapeutic value'.(E2-1.762-763) They mentioned the need for patient reassurance, which they believed depended on providing information.

\section{A relationship based on trust}

GPs interviewed in this study reported that their relationship with patients was based on trust, they were confident with patients known to the practice and they trusted patients abilities for drugs management.

Most physicians described a relationship of trust with patients: 'It is a relationship of trust that is established over time'(E7-1.358-359). The GPs were more confident with patients known to the practice: 'someone who is followed, with whom there is trust, it goes smoothly, it's not too stressful'(E12-1.187-189). They then described trusting patients who complain of pain: 'If they tell me they have pain, I believe them'(E4-1.151). In return, the patient trusts the GP and particularly values the doctor's non-judgemental approach: 'As things stand, we are quite open about what we say, I always ask them what they have, if they have taken anything, what they do not have, and so they talk, they tell me'(E2-1.573-575). One GP even mentioned a strengthening of the relationship through the management of the patient's pain: If we can have subjects for discussion other than the simple exchange from addict to GP, then so much the better. It helps to have some knowledge of the person, of his family, to treat him for other medical conditions, to discuss the weather and other aspects of life'.(E7-1.359-368)

The interviewees reported a good understanding of pharmacology by patients and trust in their abilities to manage drugs: 'They know, they can generally manage pharmacology, many of them at least know which drug to use and how to use it, in the normal way, that is'(E7-182-193).

\section{Fear of destabilising patients}

GPs interviewed in this study feared that pain and the management of pain could destabilise patients and their background treatment, and in the management, GPs integrated patients specific attitudes to drugs.

Some GPs feared destabilising the background treatment: 'I think my fear would be that they would say afterwards, 'I still have pain, I have a lot of pain', knowing that if we don't treat the cause of the pain, and if the cause is not resolved, I am afraid that we will then go from $8 \mathrm{mg}$ to 12-16 mg for weeks'(E1-1.299-303). They also reported patients' reluctance to split medication doses. They fear that they might not be able to reduce the intake frequency of OMT back to once a day after the pain has subsided: 'She says 'I could never have split my Subutex' because she would have felt that, by splitting her daily dose, she might become reliant again on something she had been fighting to be free of for a long time.'(E1-1.356-357). As a result, these GPs felt that fractionation of OMT for pain management could only be applied to stabilised patients. 'I felt that she was ready to hear that she could divide the daily dose in case of pain'(E1-1.360-361).

The physicians interviewed agreed that these patients had a specific attitude towards drugs: 'They also have a somewhat specific attitude towards drugs' (E6-1.119-120). Thus, one GP stated that the prescribed drugs were considered as a highly addictive risk by some patients: 'The drugs they are prescribed are often ultimately experienced as even more addictive, more addictive than their own substance of choice'(E5-1.553-554). They had a strong awareness of tolerance to painkillers: 'Yes, but I am addicted to drugs, I don't like taking medications anymore'(E1-1.716-717). In this context, they described frequent refusals of paracetamol.

\section{Difficulties in evaluating and treating pain}

GPs interviewed in this study stressed the importance of pain evaluation, and difficulties to assess the effectiveness of the pain management. But opinions on perception of pain in patients with OMT were divided.

Opinions about pain were divided. Thus, some GPs did not observe any specificity in pain: 'I do not get the impression that they were suffering more pain than other patients' (E8-1.29-30). Other GPs referred to their knowledge of acquired hyperalgesia, and described having observed this lowering of the pain perception threshold: 'They feel increased pain'(E1-1.671). On the other hand, the GPs mentioned a more important psychological aspect of pain: 'In terms of pain, we are just as concerned about 
understanding emotional pain, the mental component of physical pain.(E12-1.84-91). Others, on the contrary, mentioned a better tolerance of pain: That's what it says, that they could be hypersensitive to pain compared with a standard population, I admit that I haven't particularly experienced that, I have even seen some who were quite resistant to pain(E5-1.247-251).

The GPs stressed the importance of pain evaluation, some used the visual analogue scale systematically, but most stated that they used no systematic scales. They said that they used a global clinical evaluation and the functional consequences of the evaluation. Their evaluation was also based on the patient's description: 'if the patient told me he was in pain, I thought, that's it, he's in pain, if he's in a lot of pain, he's in a lot of pain'(E4-1.151). The GPs stressed the need to look for the aetiology of the pain, which guides the choice of treatment 'you need to know what the cause is if you can, (...) which will also guide the choice of analgesic' (E6-1.393-396). Most of the GPs interviewed described the effectiveness of their pain management as good: 'Well, they are in less pain. They are better, so they are happy(E8-1.180-182). Some physicians evaluated the effectiveness of pain management using a pain scale, and they re-evaluated it after treatment. However, many of them described an evaluation based on the absence of repeated requests for painkillers: 'I don't get much feedback but, if I don't see them again, I assume that is because they are fine'(E1-1.566-567).

They described difficulties in assessing the effectiveness of their management because of the lack of feedback from patients 'I rarely get feedback' (E1-1.617) and there is not enough time to see them again in consultation before too long'. 'We would have to be able to see the patient again the next day, the day after, and in current practice, that's almost impossible in town or city practices, they don't come back, or they move on'(E5-1.145-151).

\section{Difficulties for care adherence}

GPs interviewed in this study identified delays that patients with OMT experienced in their somatic management, when they suffered from medical disorders, they took a long time to seek medical help. They also underlined difficulties in compliance and due to medical nomadism.

The physicians interviewed agreed about the delays that patients were experiencing in their somatic management. They identified delays for acute pathologies: 'He broke his metatarsus, but he only came 4 days later because he was in pain'(E12-1.24-25), for dental care: 'They had to go to the dentist for 6 months'(E2-1.133-134) and for check-ups requested by their family physician: 'We make them have check-ups, most of the time they do not go for them'(E8-1.80).

The GPs interviewed spoke of patients who felt the treatment they received was holding them back: 'Patients with OMT follow-up in specialised care constantly talk, among themselves, about how they are being kept drugdependent'(E5-1.585-586). One GP mentioned that some patients adhered somewhat better to treatment than the general population. However, most of the GPs interviewed mentioned that it was difficult to obtain compliance: 'I try, of course, to ensure that they take their buprenorphine once a day in the morning and not as they need it, which is complicated'(E6-1.112-113).

They also mentioned a difficulty in maintaining a link with these patients because of cancelled appointments and medical nomadism.

Fear of misuse and diversion

GPs interviewed in this study reported fear of misuse or diversion of OMT, but they mentioned mistrust towards patients unknown to them. Self-medication was cited by GPs either as a reflect of misuse and uncontrolled intake of drugs, or as a consistent self-medication on patients stabilised with their OMT.

Regarding pain evaluation, the GPs reported the risk of prescribing to patients who are only looking for the 'high' effect of opiates: 'It is hard to make the distinction between what is real pain and what is simply a demand for.... for a product' (E12-1.111-116). Many GPs mentioned their mistrust of certain patients. They explained it as the fear of being manipulated in the context of trafficking or in misuse of OMT or analgesics. They also mentioned their mistrust towards patients unknown to them., The GPs being interviewed deplored the role of the doctor, which sometimes boiled down to merely prescribing: 'They see us as suppliers in some ways' (E8-1.82). They mentioned links between consumers in the context of trafficking or misuse of OMT: 'They gave their capsules to everyone'(E1-1.231). The physicians described difficulties arising from the misuse of several analgesic active ingredients, such as nefopam, tramadol and morphine.

The physicians interviewed described requests for immediate pain relief from patients: 'These patients are often still at the...the impetuous stage of expecting rapid results'(E6-1.430-431). In this context, they observed excessive consumption and the search for repeated and symptomatic medication in relation to opioid painkillers: ' $t$ is precisely (...) the addictive attitude that I am trying to remove with regard to medication, and that is the main difficulty'(E6-1.540-547).

Opinions were divided on the frequency of selfmedication. One GP noted an uncontrolled and indiscriminate use of products by some patients. Another mentioned a modified intake of analgesics adopted by stabilised patients: 'Those who are really well stabilised or who take small or regular doses and who are consistent (...) take analgesics more easily '(E9-1.142-144). They observed self-medication with OMT: 'Those who overuse for analgesic purposes tend not to take it, or to take it in 2 doses, morning and evening, or in case of pain that is a little more acute, they split the medication, also for analgesic purposes'(E9-1.163-168).

They mentioned guilt expressed by patients on this subject : 'I don't know if they feel guilty (...)But (...) they justify their increase in buprenorphine and they apologise for taking it (...) or try to clear themselves of the 
pain by taking buprenorphine, that's it, and they don't necessarily think about taking anything else, other than buprenorphine'(E9-1.133-140).

They went on to mention the need for a clearer framework for prescribing: 'Some patients had a rather unusual attitude towards drugs so, for these people, prescribing needs to be extremely precise' (E6-1.122-125).

\section{DISCUSSION}

In this study, GPs identified specific difficulties and limits in the management of acute pain among patients on OMT in primary care. Medical care and training challenges identified by GPs treating patients on OMT with acute pain or linked to the patient-GP relationship were identified.

\section{Place of management of acute pain among patients under OMT in primary care}

Pain is a frequent intercurrent event, destabilising the theoretical observance of OMT. ${ }^{19}{ }^{23}$ OMT management theoretically requires skills that corresponds to that at the heart of family practice. ${ }^{34}$ The management of pain among opiate-dependent patients on OMT corresponds to the theoretical central skills of general practice. GPs need to be involved in patient follow-up, to improve access to OMT, in patient-centred programme, as underlined by European consensus. ${ }^{28}$ Two-thirds of OMT are prescribed by GPs in France, ${ }^{35}$ and in a population of patients on OMT in primary care, over $25 \%$ have associated somatic disorders. ${ }^{36}$ But in the present study, the GPs reported that it was a rare situation, and that this situation did not correspond to their professional skills. They reported the lack of training, and the lack of time in their daily practice and the need to address patients with an OMT and acute pain to specialised facilities. These findings correspond to the deficiencies identified in the literature, where very few articles have dealt with the management of pain among patients on OMT, where the majority are only concerned with MTD. ${ }^{37}$ But France is an exception in allowing buprenorphine to be prescribed by any practitioner, ${ }^{7}$ and therefore GPs have to face the management of longterm buprenorphine treatment, including management of pain among patients on buprenorphine. ${ }^{47}$ These findings could also be set against the specific place of OMT management, independently from the acute pain event. OMT are considered as a type of treatment unlike other treatments by a large majority of patients and also by physicians and pharmacists. ${ }^{21}$ A minority of early-career family physicians report having received adequate preparation to provide buprenorphine treatment during their residency. ${ }^{4}$ However, a recent study showed that currently primary care providers seemed less engaged in BHD initiation among OUD patients, while pharmacists have not modified their involvement towards patients under BHD treatment. $^{38}$

Nevertheless, in our study, the GPs were aware of the problem of acute pain among opioid-dependent patients and were concerned about the specificities in this patient population. They also underlined that patients on OMT presented difficulties in accessing care for acute and painful disorders (dental, traumatic, etc). Indeed the proportion of patients on OMT not asking for help when faced with pain is high: compared with $28 \%$ in the general population, when experiencing pain, $40 \%$ of patients on OMT did not ask for help. ${ }^{39}$ In this sample, the GPs identified patients who had a specific perception of their own bodies; they identified the care required for patients on OMT, for patients with hyperalgesia and also for those patients who had greater tolerance of painful symptoms.

The GPs in our study reported a global individualised patient-centred approach to acute pain among patients on OMT, they described a global evaluation of patients' pain, and an individualised approach integrating age, the psychopathological profile and also sensitivity to pain and patient perceptions of their bodies. The GPs used a global clinical evaluation including the functional consequences of pain. Their evaluation was also based on patients' descriptions of the types of pain experienced and it distinguished between the psychological and nociceptive components of pain. This is in line with the literature, reporting that $94 \%$ of primary care physicians relied mainly on questioning patients to evaluate pain. ${ }^{40}$

The GPs underlined difficulties in evaluating and treating pain for patients on OMT, and difficulties in reassessing pain and pain treatment strategies. They were also concerned because they apprehended the risk of patient destabilisation, and the fear felt by patients regarding this destabilisation. These risks are real, as the risk of pain being undertreated is significantly high among opioiddependent patients ${ }^{41}$ and acute pain that is not sufficiently relieved exposes the patient to a 2.3 times higher risk of premature discontinuation of treatment. ${ }^{42}$

Moreover, what appeared clearly as a subtheme was that the doctor-patient relationship was based on trust. GPs reported a relationship based on trust with patients whom they knew, and they even described trust in patient know-how regarding the management of drugs. One GP even mentioned developing a closer relationship with the patient through management of the patient's pain, with an evaluation centred on the patient, including variables not directly linked to opioid dependence.

This reported trust differs from the findings by Quinlan and Cox,${ }^{24}$ where mistrust was high among professionals faced with patients on OMT and hospitalised. Mistrust on the part of doctors, linked to fear of misuse or diversion, was also cited in our study, and concerned more particularly situations where the patients were not known to the practitioner. Mistrust of certain patients and a fear of being manipulated in the trafficking or misuse of OMT or analgesics were described. These fears and negative representations probably hinder medical reasoning and reduce the effectiveness of management. ${ }^{22} 4344$ But, despite drug regulations, GPs did not mention the fear of inspections or regulatory measures. Fear of misuse or diversion was associated by GPs with some painkillers, 
or with patient networks and with patients they did not know.

\section{Lack of guidelines on pharmacotherapy for treating acute pain among patients with OMT}

The feeling of a lack of references or guidelines, or the lack of knowledge as to what does exist, was a major subtheme in this study. The GPs stated that decisions about prescribing were based on empiricism rather than protocols, which probably induces interpersonal variability in pain management, as reported in a study conducted in an A\&E department. ${ }^{45}$

Little consensus exists in the literature about the management of acute pain among opiate-dependent patients on OMT, 2254 and certainly none specifically in general practice. Most of these recommendations agree on avoiding the prescription of weak opioid analgesics and on the interest in using non-narcotic medication in first resort (non-steroid anti-inflammatory drugs, antidepressants, anxiolytics...), as well as on the necessity of psychosocial support. ${ }^{2425}$ In our study, the GPs remarked on the psychological component of pain in patients under OMT and the need for psychological support.

Regarding pharmacotherapy, weak opioids, defined by GPs as level 2 painkillers (WHO) were widely prescribed by the sample, in particular paracetamol+codeine. This prescription may seem surprising from GPs skilled in prescribing OMT as defined in this study. The combination of level 2 painkillers (WHO) with MTD is not recommended either, because it is not effective. ${ }^{46} 47$ For those on BHD, there is limited evidence to support the safety of adding an opioid agonist while continuing BHD. ${ }^{25} 49$ However, as reported by GPs in our study, patients prescribed BHD often continue to receive prescriptions for other opioid medications. An analysis of patients initiating BHD across 11 states in the USA $(n=38096)$ found that $43.2 \%$ of patients received at least one dispensation of another opioid medication during the treatment episode. ${ }^{50}$ In family practice, $50 \%$ of GPs reported using a level 2 analgesic prescription combined with OMT, ${ }^{51}$ and in emergency department $19 \%$ of physicians reported prescribing level 2 analgesics combined with OMT, ${ }^{42}$ which is comparable with another study. ${ }^{45}$ A 2-year follow-up of 1182 patients on OMT also found that the most widely prescribed analgesic, after nonsteroid anti-inflammatory drugs, was a combination of paracetamol+codeine, representing $9 \%$ of the analgesics prescribed. ${ }^{52}$

Among the weak opioids, tramadol was cited in our study. It has a specific pharmacological profile, involving both opioid-receptor and serotonergic action that provides a theoretical mechanism for effective analgesic effect when used concomitantly with BHD. ${ }^{49}$ Donovan $e t$ $a l^{49}$ showed in a national survey that $6.4 \%$ of all patients using BHD/NX had at least one overlapping prescription for tramadol during the study. But it was not specifically cited for its efficacy in our study, on the contrary, it was identified as an opioid potentially at risk for misuse or trafficking.

Regarding the ASAM practice guidelines, when OMT is prescribed, for the management of pain OMT dosage should be maintained or increased on a temporary basis and administered in a staggered schedule rather than in a single daily dose. ${ }^{25}$ In case of severe pain, recommended pharmacological strategies differ between MTD and BHD or BHD/NX. ${ }^{2425} 2753$ In our study, the GPs did not differ in attitudes between MTD and BHD and BHD was more often cited as a concern than MTD, which is consistent with the French prescribing model for OMT and BHD. ${ }^{28}$ Regarding the recommended staggered schedule, GPs did not seem at ease with this strategy, and feared patient destabilisation. They reported fear of destabilisation and of poor compliance with treatment, so that they preferred to add another analgesic rather than increasing and dividing OMT dosage during the day. This is in line with previous work, where $41 \%$ of the GPs questioned were familiar with the possibility of using OMT for its analgesic effect, but only $43 \%$ of them actually used it for analgesic purposes. The reason given by physicians for not prescribing was fear of uncontrolled return to OMT consumption. ${ }^{40}$

The GPs interviewed here had a global approach to the management of their patients, whom they were monitoring both for addictive disorders and for medical follow-up. In terms of addictive disorders, they described how they countered the tendency of many patients to split OMT intake. They felt that asking the patient to split his or her treatment for the alleviation of pain was inconsistent with their previous discussions. They preferred to prescribe a second active ingredient to clarify the temporary aspect and indications of this treatment.

They also integrated the patient point of view, and described reluctance on the part of the patients, some patients were not (or did not feel) sufficiently stabilised to have the ability to split the MSO without slipping into uncontrolled consumption. However, the GPs cited the fact that some patients used OMT for analgesic purposes on their own initiative, but it was considered as a misuse, and was associated with guilty feelings on the part of patients. One study showed that $61 \%$ of the patients on OMT were aware of the staggered schedule of OMT for analgesic purposes, a majority $(68 \%)$ considered it effective and $58 \%$ of them used it regularly. ${ }^{40}$

While it is stated that existing pain management protocols are not widely applied, ${ }^{19}{ }^{27}$ the factors limiting application were not well known, and this applied particularly in general practice. In our study, the prescription of weak opioid painkillers reflected a certain empiricism in a situation identified as quite rare, complex to evaluate and lacking a reference recommendations. Despite pharmacological interactions and risks, physicians interviewed in this study reported their feelings on the analgesic efficacy of codeine in combination with buprenorphine and/or methadone. However, among patients on OMT, opioid analgesic treatment exposes patients to an increased risk 
of side effects, misuse, problems of tolerance and dependence. ${ }^{41} 4254$ In all events, the guidelines have shown many limits regarding opioids prescriptions more widely. As an example, in the USA, despite a major opioid crisis, most clinicians do not follow best practices for opioid prescribing, despite national guidelines and educational programmes. ${ }^{55}$ One hypothesis is that the guidelines most of the time focus on changes in individual prescribing habits.

\section{Lack of care coordination and networking support for clinicians treating acute pain in patients with OMT}

The development of a multidisciplinary network, perceived as a need by the GPs in this study, could be an interesting back-up for acute pain management among patients on OMT. GPs in this study described relationship difficulties as a limiting factor in professional networks, between GPs and pharmacists, and also hospitals, for different reasons: lack of communication and patient information regarding pain management, and also negative views and stigmatisation of patients on OMT by professional networks. Good communication with primary care clinicians, dispensing pharmacists, and addiction treatment facilities is essential to ensure that patients re-engage with supporting services and maintain their pre-hospital management. ${ }^{24}$ In addition, more globally, regarding opioid prescription, Quinlan et $a \varphi^{\tilde{7}}$ noted the role of primary care prescribers in opioid prescriptions: they are, compared with those in hospitals, in a better position to recognise patients at higher risk of developing problem opioid use, when there are existing mental health issues or substance misuse.

The GPs did not mention nurses in their potential networks. The French model of OMT issue and buprenorphine prescription is centred on medical prescription and management in collaboration with pharmacists. ${ }^{7}$ The nurse care manager model developed in officebased buprenorphine treatment for opioid use disorders in the USA has shown its efficacy in risk management and monitoring of OMT prescribing. ${ }^{58}{ }^{59}$ Nurse care managers can use a stepped-care approach, combining cognitive behavioural therapy methods with medication and improvement of pain-related disability, in collaboration with GPs. The development of primary care multiprofessional centres in France, including nurses, could probably help to improve the management of acute pain among patients on OMT.

\section{Strengths and limitations}

In the literature, there are very few articles on this topic. The qualitative method helped us to obtain in-depth answers about the management of acute pain among patients on OMT. The GPs interviewed described their clinical practice along with patient narratives, and sometimes recounted failure to manage pain among patients on OMT. This openness during the interviews confirmed that the atmosphere created by the interviewers was appropriate. The data was based on real life experiences and not on general opinions.

This study has several limitations. First, we selected a purposive sample using a snowball method. The first three GPs were identified by the research committee and the nine other GPs were selected using a snow ball method. The sample comprised GPs with experience in OMT prescription, either MTD or BHD, but none of the physicians interviewed was a prescriber of High Dosage Buprenorphine- Naloxone (BHD-NX). We realise that our study involved only a small selected number of GPs, who were experienced in OMT prescription, and who came from the same region in France. The sample of GPs interviewed consisted of a majority of males in urban practices. A majority had a long practice of medicine (8 GPs interviewed had more than 20 years' experience). Young physicians, women, and GPs in rural practices were lacking in this sample. This limits transferability. But despite the fact that in France since 1996, the regulatory prescribing framework has been extended to include any physician, the population of physicians implicated in OMT management is specific. In a previous study we showed that only $42 \%$ of independent GPs prescribed buprenorphine as a first-line prescription, and 40\% of GPs were not following up any patient on buprenorphine. ${ }^{4}$ Another limitation is that some GPs answered too briefly, 5 interviews lasted less than $20 \mathrm{~min}$. This could be explained by the specificities of primary care consultations: a study in 2019 in France showed that GPs reported a median duration of consultation of $18 \mathrm{~min}$, and $40 \%$ reported less than $15 \mathrm{~min}$, and men declared shorter durations than women. ${ }^{60}$

Participants did not review or provide feedback on the findings. Theoretical saturation was obtained at the eighth interview. The interview guide was short, and focused on the specific research question. The guide was not modified during the study, and even if an interview was short, the content was found informative by the researchers in relation to the research question. The rarity and specificity of the situation explored may have contributed to this early saturation. Finally, the data was collected in 2015, which is also a limitation. But no changes in policy or recommended practice in OMT have appeared since the data was collected, and our results are informative in the setting of the paucity of existing data in literature.

\section{CONCLUSION}

The complexity of acute pain and opioid dependence represents significant challenges for clinicians and patients, both regarding medical skills and regarding the patient-GP relationship. In primary care, it is hard to achieve a balance between pain relief and OUD treatment, in a global patient-centred approach. Fear of misuse or diversion by the patient was not a major issue, except for patient not known to the practitioner, but GPs were concerned with the risks of patient destabilisation in acute pain situations. A perceived lack of medical training on the subject, and a lack of guidance for these situations 
were highlighted in the study. To limit inconsistencies in information given to patients by the various stakeholders, training should be provided targeting a multidisciplinary population (private or hospital doctors, family physicians, specialists, pharmacists, nurses, etc), forming a multidisciplinary network which seems important to improve patient care. The willingness to work in a network was unanimous among the GPs interviewed. This mode of functioning makes it possible to obtain advice and a procedure to be followed rapidly, when the family physician is faced with a difficult situation. Initially, it could be necessary to organise targeted training for the most experienced OMT prescribers. These GPs, who are often the main supporters of informal networks in liberal medicine, could thus facilitate the dissemination of this training. Existing protocols do not seem to be in line with general practice. However, obtaining clear recommendations for care will be increasingly important in the future. Indeed, the number of patients on OMT has increased since it was first marketed, and these patients experience more pain than the general population. GPs will increasingly have to deal with these situations and will have to develop their own guidelines. It will, therefore, be valuable to evaluate the prescribing practices of family physicians and their effectiveness on pain, as well as the sustainability of substitution, using quantitative methods.

\section{Acknowledgements Many thanks for all the GPs who agreed to participate in the study.}

Contributors MGL, JYLR and MB designed the study, MB collected data, MB and MGL analysed qualitative data, JYLR and DLG participated to the redaction and reviewed the article.

Funding The authors have not declared a specific grant for this research from any funding agency in the public, commercial or not-for-profit sectors.

Competing interests None declared.

Patient and public involvement Patients and/or the public were not involved in the design, or conduct, or reporting, or dissemination plans of this research.

Patient consent for publication Not required.

Ethics approval This study was approved by the ethics committee of the 'Université de Bretagne Occidentale'.

Provenance and peer review Not commissioned; externally peer reviewed.

Data availability statement All data relevant to the study are included in the article or uploaded as online supplemental information.

Open access This is an open access article distributed in accordance with the Creative Commons Attribution Non Commercial (CC BY-NC 4.0) license, which permits others to distribute, remix, adapt, build upon this work non-commercially, and license their derivative works on different terms, provided the original work is properly cited, appropriate credit is given, any changes made indicated, and the use is non-commercial. See: http://creativecommons.org/licenses/by-nc/4.0/.

ORCID iD

Morgane Guillou Landreat http://orcid.org/0000-0003-1568-9480

\section{REFERENCES}

1 Degenhardt L, Whiteford HA, Ferrari AJ, et al. Global burden of disease attributable to illicit drug use and dependence: findings from the global burden of disease study 2010. Lancet 2013;382:1564-74.

2 American Psychiatric Association. Diagnostic and statistical manual of mental disorders. 5th ed. Washington, DC: American psychiatric Association, 2013.
3 Guillou Landreat M, Victorri-Vigneau C, Grall-Bronnec M. Impact des politiques de santé publique sur les consultations en addictologie partir d'un suivi longitudinal de 1998 2007. Ann Med Psy 2013;171:367-71.

4 Guillou Landreat M, Rozaire C, Guillet Jyves, et al. French Experience with Buprenorphine : Do Physicians Follow the Guidelines? PLoS One 2015;10:e0137708.

5 Rolland B, Bouhassira D, Authier N, et al. [Misuse and dependence on prescription opioids: Prevention, identification and treatment]. Rev Med Interne 2017;38:539-46.

6 Guillou Landreat M, Sebille-Rivain V, Victorri Vigneau C, et al. Buprenorphine prescription compliance: an original observational and longitudinal study. J Psychoactive Drugs 2014;46:162-7.

7 Fatseas M, Auriacombe M. Why buprenorphine is so successful in treating opiate addiction in France. Curr Psychiatry Rep 2007;9:358-64.

8 OFDT. Baromètre Santé 2017 Paris 2018. Available: https://www. ofdt.fr/statistiques-et-infographie/sources-statistiques/barometresante-alcool/

9 Zhang L, Zhang D, Chen W, et al. High prevalence of HIV, HCV and tuberculosis and associated risk behaviours among new entrants of methadone maintenance treatment clinics in Guangdong Province, China. PLoS One 2013;8:e76931.

10 Klepser ME, Klepser TB. Drug treatment of HIV-related opportunistic infections. Drugs 1997;53:40-73.

11 Sulkowski MS, Mast EE, Seeff LB, et al. Hepatitis C virus infection as an opportunistic disease in persons infected with human immunodeficiency virus. Clin Infect Dis 2000;30 Suppl 1:S77-84.

12 Basu D, Banerjee A, Harish T, et al. Disproportionately high rate of epileptic seizure in patients abusing dextropropoxyphene. Am J Addict 2009;18:417-21.

13 Manninen PH. Opioids and seizures. Can J Anaesth 1997;44:463-6.

14 Jovanović-Čupić V, Martinović Žarko, Nešić N. Seizures associated with intoxication and abuse of tramadol. Clin Toxicol 2006;44:143-6.

15 Mattoo SK, Singh SM, Bhardwaj R, et al. Prevalence and correlates of epileptic seizure in substance-abusing subjects. Psychiatry Clin Neurosci 2009;63:580-2.

16 Amari E, Rehm J, Goldner E, et al. Nonmedical prescription opioid use and mental health and pain comorbidities: a narrative review. Can J Psychiatry 2011;56:495-502.

17 Kobus AM, Smith DH, Morasco BJ, et al. Correlates of higher-dose opioid medication use for low back pain in primary care. $J$ Pain 2012;13:1131-8.

18 Fridell M, Bäckström M, Hesse M, et al. Prediction of psychiatric comorbidity on premature death in a cohort of patients with substance use disorders: a 42-year follow-up. BMC Psychiatry 2019;19:150

19 Courty P, Authier N. [Pain in patients with opiates dependence]. Presse Med 2012;41:1221-5.

20 Yi P, Pryzbylkowski P. Opioid induced hyperalgesia. Pain Med 2015;16:S32-6.

21 Higgins C, Smith BH, Matthews K. Evidence of opioid-induced hyperalgesia in clinical populations after chronic opioid exposure: a systematic review and meta-analysis. $\mathrm{Br} \mathrm{J}$ Anaesth 2019;122:e114-26.

22 Alford DP, Compton P, Samet JH. Acute pain management for patients receiving maintenance methadone or buprenorphine therapy. Ann Intern Med 2006;144:127-34.

23 Delorme J, Chenaf C, Bertin C, et al. Chronic pain Opioid-Maintained patients receive less analgesic opioid prescriptions. Front Psychiatry 2018;9:335.

24 Quinlan J, Cox F. Acute pain management in patients with drug dependence syndrome. Pain Rep 2017;2:e611.

25 Kampman K, Jarvis M. American Society of addiction medicine (ASAM) national practice guideline for the use of medications in the treatment of addiction involving opioid use. J Addict Med 2015;9:358-67.

26 Huxtable CA, Roberts LJ, Somogyi AA, et al. Acute pain management in opioid-tolerant patients: a growing challenge. Anaesth Intensive Care 2011;39:804-23.

27 Mehta V, Langford R. Acute pain management in opioid dependent patients. Rev Pain 2009;3:10-14.

28 Dematteis M, Auriacombe M, D'Agnone O, et al. Recommendations for buprenorphine and methadone therapy in opioid use disorder: a European consensus. Expert Opin Pharmacother 2017;18:1987-99.

29 Mays N, Pope C. Qualitative research in health care. Assessing quality in qualitative research. BMJ 2000;320:50-2.

30 Pope C, van Royen P, Baker R. Qualitative methods in research on healthcare quality. Qual Saf Health Care 2002;11:148-52.

31 Green J, Thorogood N. Qualitative methods for health research. London: Sage, 2009. 
32 Braun V, Clarke V. Using thematic analysis in psychology. Qualitative Research in Psychology 2006;3:77-101.

33 Antoine P, Alan Smith J. Saisir l'expérience : présentation de l'analyse phénoménologique interprétative comme méthodologie qualitative en psychologie. Psychologie Française 2017;62.

34 Loxterkamp D. Medication-Assisted treatment should be part of every family physician's practice: Yes. Ann Fam Med 2017;15:309-10.

35 OFDT. Drogues et addictions, données essentielles.

36 Gentile G, Frauger E, Giocanti A, et al. [Characteristics of subjects under opiate maintenance treatment in primary care using the OPEMA data 2013]. Therapie 2016;71:307-13.

37 Eyler ECH. Chronic and acute pain and pain management for patients in methadone maintenance treatment. Am J Addict 2013;22:75-83.

38 Dupouy J, Maumus-Robert S, Mansiaux Y, et al. Primary Care of Opioid use Disorder: The End of "the French Model"? Eur Addict Res 2020;26:346-54.

39 Douleur VF. Souffrance et toxicomanie : évaluation et traitement de la douleur chez le patient sous traitement de substitution enquête dans le loiret en 1999 Tours: Université François Rabelais 1999.

40 Lefevre-Mohr C. Quelle prise en soins du patient douloureux sous traitement de substituion aux opiacés ? analyse des comportements d'automédication des patients et despratiques antalgiques des médecins Strasbourg University of Strasbourg 2014.

41 Scimeca MM, Savage SR, Portenoy R, et al. Treatment of pain in methadone-maintained patients. Mt Sinai J Med 2000;67:412-22.

42 Bounes V, Jouanjus E, Roussin A. Acute pain management for patients under opioid maintenance treatment : what physicians do in emergency department ? Eur J Emerg Med 2013;44:127-34.

43 Newshan G. Pain management in the addicted patient. Nurse Pract 2000;25:7-18.

44 Guillou Landreat M. Représentation des traitements de substitution aux opiacés et de leur arrêt. Regards croisés patients-médecins 2013:23-44.

45 Desplas M. Prise en chargde de la douleur chez le suejt sous médicament de substituion Toulouse: Université de Toulous 2005.

46 Victorri-Vigneau C, Bronnec M, Guillou M, et al. Prise en charge de la douleur aiguë CheZ les patients sous traitements de substitution aux opiacés. Douleur et Analgésie 2012;25:83-6.

47 Laprevote V, Geoffroy PA, Rolland B, et al. [Management of opioid maintenance treatments when analgesic treatments are required]. Presse Med 2013;42:1085-90.
48 Olivier M, Roussin A, Bayle P. Prise en charge des douleurs faibles modér\&es et des douleurs fortes de patients substaitués pas buprénorphine ou méthadone pour une pharmacodépendance majeure aux opiacés France CLUD 2013;39.

49 Donovan K, Kogut S, Buchanan A, et al. A claims analysis of the utilization of tramadol for acute pain in patients prescribed buprenorphine/naloxone for opioid use disorder. J Opioid Manag 2018:14:407-13.

50 Daubresse M, Saloner B, Pollack HA, et al. Non-buprenorphine opioid utilization among patients using buprenorphine. Addiction 2017;112:1045-53

51 Dumand E. Prise en charge en médecine générale d'une douleur aigue chez un patient toxicomane aux opiacés Grenoble université de Grenoble 2008.

52 Fredheim OMS, Borchgrevink PC, Nordstrand B, et al. Prescription of analgesics to patients in opioid maintenance therapy: a pharmacoepidemiological study. Drug Alcohol Depend 2011;116:158-62.

53 Kampman K, Jarvis M, Comer S. American Society of addiction medicine (ASAM) national practice guideline for the use of medications in the treatment of addiction involving opioid use. $J$ Addict Med 2015;9:358-67.

54 Heiskanen T, Kalso E. Non-Analgesic effects of opioids: interactions between opioids and other drugs. Curr Pharm Des 2012;18:6079-89.

55 Morasco BJ, Duckart JP, Dobscha SK. Adherence to clinical guidelines for opioid therapy for chronic pain in patients with substance use disorder. J Gen Intern Med 2011;26:965-71.

56 Liebschutz JM, Xuan Z, Shanahan CW, et al. Improving adherence to long-term opioid therapy guidelines to reduce opioid misuse in primary care: a cluster-randomized clinical trial. JAMA Intern Med 2017; 177:1265-72.

57 Quinlan J, Rann S, Bastable R, et al. Perioperative opioid use and misuse. Clin Med 2019;19:441-5

58 Alford DP, LaBelle CT, Kretsch N, et al. Collaborative care of opioidaddicted patients in primary care using buprenorphine: five-year experience. Arch Intern Med 2011;171:425-31.

59 LaBelle CT, Han SC, Bergeron A, et al. Office-Based opioid treatment with buprenorphine (OBOT-B): statewide implementation of the Massachusetts collaborative care model in community health centers. J Subst Abuse Treat 2016:60:6-13.

60 Chaput H, Monziols M, Fressard L. Deux tiers des médecins généralistes libéaux déclarent travailler au moins 50 heures par semaine. Etudes et Resultats. DREES 2019:1113. 